\title{
Research on Design Strategies of Cognitive Training System Based on SCD Elderly Behavioral and Psychological Characteristics
}

\author{
XUE Yanmin ${ }^{1}$, LIN Rui ${ }^{2}$, GE Chang ${ }^{3}$ \\ School of Art and Design, Xi 'an University of Technology, Xi ' an, Shaanxi Province, China, 710054
}

\begin{abstract}
Alzheimer's disease (hereinafter referred to as AD) has become a major global health problem for the elderly. Combining with the current computer-aided design system design for prevention and training of Alzheimer's disease is one of the important ways to solve this problem. This study aims at the early subjective cognitive decline of Alzheimer's disease (hereinafter referred to as SCD). Through investigation and research, the behavior and psychological characteristics of SCD elderly are sorted out, and the main functions of cognitive training system are located. The main functions, interactions, colors and layout of the current cognitive system are summarized by analyzing the existing cognitive training system, and compared with the research results, a more reasonable system function, interaction mode, interface color and layout are determined. Therefore, the cognitive training system design strategy based on the behavioral and psychological characteristics of SCD elderly is summarized.
\end{abstract}

\section{Introduction}

Population aging has become a serious social problem in our country. With the aging and degeneration of the physical functions of the elderly, the health problems brought by aging are also increasing. Among the 130 million elderly population in our country, the number of the elderly suffering from $\mathrm{AD}$ is more than 6 million, currently ranking first in the world ${ }^{[1]}$. AD is irreversible and incurable. It can only be intervened by the combination of drug therapy and non-drug therapy to delay the development of the disease.

$\mathrm{AD}$ is a continuous pathophysiological process, which has gone through three stages: subjective memory decline stage(SCD), mild cognitive impairment stage(MCI) and $\mathrm{AD}$ dementia stage ${ }^{[2]}$. SCD is considered to be the initial stage of $\mathrm{AD}$ onset. The incidence rate for the elderly over 65 years old is $25 \%-50 \%$, and $\mathrm{MCI}$ is expected to occur in about 15 years ${ }^{[3]}$. The incidence rate of MCI for the aged $60-78$ is $12.7 \%$, while about $50 \%$ of MCI patients will develop dementia within 5 years ${ }^{[4]}$.

The earliest description of SCD symptoms is Reisberg et $\mathrm{al}^{[3]}$. proposed to divide cognitive impairment into 7 stages. The very slight cognitive decline in the second stage is consistent with the description of SCD. The concept of SCD was put forward in 2012. This concept has undergone many definitions, causing a lot of inconvenience to the research. Professor Frank Jessen first confirmed the concept of SCD in Alzheimer's \& Dementia magazine in $2014^{[5]}$.

Due to the irreversibility and incurable nature of $\mathrm{AD}$, intervention on $\mathrm{AD}$ by means of a series of cognitive training and drug therapy can only play the role of prevention and delay. Clare [6] divides cognitive intervention into three different concepts: (1) General cognitive stimulation; Intervention activities are carried out by general methods to improve the cognitive and social functions of the target population. (2) Cognitive training; Guiding training for specific aspects of cognition (such as memory, language, attention or executive ability.). (3) Intervention programs for specific daily activities are aimed at improving practical difficulties related to daily activities.

Reisberg etc. found that SCD is the most primitive stage of AD. As a high-risk factor that may lead to AD, effective implementation of early intervention is of great significance to prevent and delay the decline of cognitive function and the AD process. With SCD elderly as the intervention target, especially non-drug intervention programs have attracted more and more interest in dementia prevention researchers. SCD does not recommend drugs. Currently, mainstream non-drug intervention methods include: cognitive training, cognitive rehabilitation, etc. ${ }^{[7]}$, as well as non-drug interventions that do not directly target cognitive functions, such as physical exercise, supplementary nutrition, supplementary/alternative medical interventions (such as meditation, yoga, etc. $)^{[8]}$. In recent years, the use of cognitive training to improve memory decline of patients has received much attention, but the research on cognitive training for SCD elderly is still in its infancy. 


\section{Existing Research on Cognitive Training}

So far, the commonly used cognitive training methods at home and abroad are memory training, reasoning training, processing speed training, strategy training, psychomotor training ${ }^{[9]}$,etc. There is also comprehensive cognitive training that uses two or more methods simultaneously. The type of cognitive impairment (as shown in Fig.1) determines the difference in cognitive training. Each type of cognitive impairment has corresponding cognitive training.

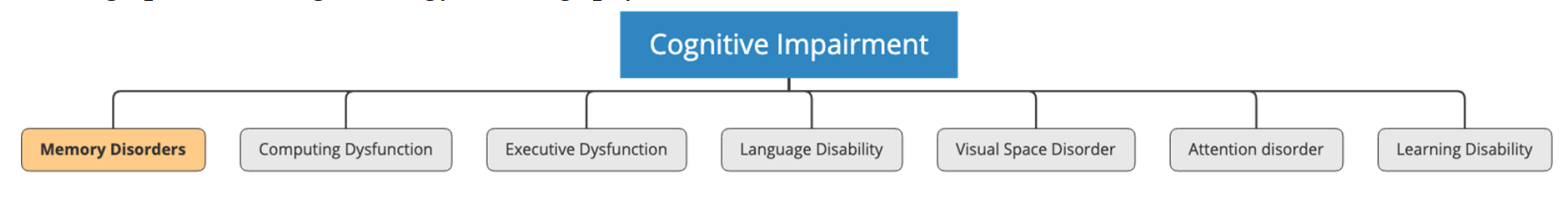

Fig. 1 Type of cognitive impairment.

Each training has its own specific method to adapt to it $^{[10]}$. (as shown in Tab. 1):

Tab. 1 Training method for cognitive impairment.

\begin{tabular}{|c|c|}
\hline Name & Methods \\
\hline \multirow{4}{*}{$\begin{array}{l}\text { Strengthen } \\
\text { Memory } \\
\text { Training }\end{array}$} & Graphic Recognition Method; \\
\hline & Information Repetitive Memory \\
\hline & Method; Image-Text Combination \\
\hline & Memory Method \\
\hline Time & According to the situation to re-plan \\
\hline Concept & the patient's work and rest time (what \\
\hline Training & concept of time \\
\hline \multirow{3}{*}{$\begin{array}{l}\text { Language } \\
\text { Training }\end{array}$} & $\begin{array}{l}\text { Things retelling training (formulating } \\
\text { tasks such as reading and retelling }\end{array}$ \\
\hline & content) Reading pictures to \\
\hline & $\begin{array}{l}\text { understand pictures and reciting } \\
\text { ancient poems and articles }\end{array}$ \\
\hline $\begin{array}{l}\text { Arithmetic } \\
\text { Ability } \\
\text { Training }\end{array}$ & $\begin{array}{l}\text { Simple arithmetic problems, including } \\
\text { daily reckoning training }\end{array}$ \\
\hline \multirow{4}{*}{$\begin{array}{l}\text { Attention } \\
\text { Training }\end{array}$} & Finger guessing game, music \\
\hline & appreciation, finding different games, \\
\hline & edify sentiment, but also improve \\
\hline & concentration \\
\hline \multirow{3}{*}{$\begin{array}{l}\text { Reasoning } \\
\text { Strategy } \\
\text { Training }\end{array}$} & \\
\hline & Study the laws between words and \\
\hline & \\
\hline
\end{tabular}

With the development of computer-aided design, in the treatment of cognitive disorders, the use of computers for patients' orientation, visual perception, spatial perception, movement application, visual motor organization, thinking action, attention and other aspects of training have gradually become clinical Simple and easy treatment. Zhu Lin etc ${ }^{[1]}$,Wang Xiaona etc ${ }^{[12]}$ Selected patients with brain impairment with cognitive dysfunction and depressive symptoms to conduct traditional rehabilitation training and computer-aided cognitive training experiments, respectively, both achieving better results than traditional rehabilitation. Cognitive training software has also shown its advantages. The Basic Cognitive Ability Test (version 2.0) and its software, designed and compiled by Chinese Academy of Sciences, have the advantages of being objective, accurate, effective, and convenient $^{[13]}$.

\section{Behavioral and Psychological Characteristics of SCD Elderly}

According to the results of consulting relevant medical materials and interviews with experts from Tang Du Hospital in $\mathrm{Xi}$ 'an City, Shaanxi Province, the basic physiological characteristics of elderly patients are analyzed. SCD is more common in the middle-aged and elderly people, which can occur in healthy people with cognitive decline without objective evidence. The incidence rate of SCD in the elderly is $25 \%-88 \%$, and the incidence rate increases with age: the incidence rate is $20 \%$ when the age is less than 65 years old, $25 \%-50 \%$ when the age is no less than 65 years old, and $88 \%$ when the age over 85 years old ${ }^{[14]}$.

\subsection{Sample Research}

According to the particularity of SCD in the elderly, onsite observation interviews and later analysis and summary are adopted to understand the exact condition of SCD. Through on-site interviews with doctors, caregivers and patients themselves, the required data can be obtained. The main purposes of the interview are as follows: (1) The basic information of SCD patients and general situation of their daily living ability. (2) The treatment methods of hospitals, doctors and nurses, as well as the matters needing attention in the later stage of patients. (3) A preliminary understanding of the troubles encountered during illness can be obtained by asking family members and patients themselves. (4) The patients' interests and hobbies by asking their families and patients themselves. (5) The patient's family background by asking caregivers.

The survey was conducted in the Department of Neurology, Tang Du Hospital, Fourth Military Medical University. The survey was conducted between May 8 and September 10, 2019, with 30 patients, 30 family members and 4 doctors. Because the patient has memory impairment and the patient's chief complaint also has deviation, the research mainly focuses on interviewing caregivers. The following is the degree of cognitive decline of the respondents (Table 2). 
Tab. 2 Number of Cognitive Impairment Declines.

\begin{tabular}{|c|c|c|c|}
\hline \multirow{3}{*}{$\begin{array}{c}\text { Types of } \\
\text { cognitive } \\
\text { impairment } \\
\text { Memory }\end{array}$} & \multicolumn{3}{|c|}{ Degree of decline (number of people) } \\
\hline & Strong & Medium & Weak \\
\hline & 6 & 13 & 11 \\
\hline $\begin{array}{l}\text { Language } \\
\text { ability }\end{array}$ & 4 & 12 & 14 \\
\hline Attention & 3 & 14 & 13 \\
\hline $\begin{array}{l}\text { Arithmetic } \\
\text { ability }\end{array}$ & 2 & 9 & 19 \\
\hline $\begin{array}{l}\text { Visual } \\
\text { space } \\
\text { ability }\end{array}$ & 6 & 11 & 13 \\
\hline
\end{tabular}

\subsection{Analysis of Behavioral and Psychological Characteristics}

Behavior and psychology are inseparable. Due to psychological cognitive impairment, SCD elderly people show obvious characteristics in the following aspects:

\subsubsection{Memory and Computational Ability Decline.It} can be seen from the research samples that the memory decline of patients is worse than that of normal aging. When dealing with complex things, they are often unable to start and slow to respond. It is also due to the memory decline, unable to continue the hobby before the onset. These symptoms slowly begin to affect the daily life of patients. It is usually manifested by forgetting where things are placed, not being able to name or not being fully familiar with people's names, forgetting the next schedule, etc.

Compared with normal aging, the calculation ability decreases significantly, the sensitivity to numbers decreases, and the simple calculation ability is insufficient.

\subsubsection{Visual Space Disorder.Another common point in} the survey sample is the unclear direction. However, patients can return home alone in familiar places and complete daily activities such as shopping and cooking. Once the distance exceeds, the patient will lose his direction and need someone to accompany him. Because the patient's spatial orientation is weakened. Some elderly patients choose not to go out for fear of losing their direction, resulting in a lack of exercise and social activities.

\subsubsection{Decrease in Attention and Executive} Ability.Caregivers reflect that patients are often accompanied by drowsiness, staring blankly at TV, etc. Elderly patients have a significant decline in attention compared with normal elderly of the same age. Due to uneven distribution of attention, the speed of transaction processing and sustained attention have different degrees of decline, and the time spent in transaction processing is more than usual.

As the speed of transaction processing is slower than that of normal peers, the execution ability will also be affected, and the order of transaction processing will be more ambiguous than that of normal old people. When patients deal with relatively complicated tasks and encounter new situations, the problem of executive function decline occurs frequently in the process of learning new skills. Learning is slow and easy to forget. Caregivers need more patience.

3.2.4 High Incidence of Mental IIIness. SCD is not only manifested in the decline of cognitive function, but also affects psychology and spirit. In the early stage of AD, the elderly has the most and most complicated psychological changes, such as depression, irritability, anxiety, apathy and even aggressive behaviors. Often for their own brain diseases, memory loss, severe insomnia and other physical and mental problems and very distressed, and then sad depression, loss of confidence in life. According to the survey samples, the patients have changed their temperament greatly after the onset of the disease, lack confidence, and choose not to go out for fear of getting lost. The negative psychology of inferiority prevents the patients from participating in social activities and from continuing their previous hobbies. Some even remain silent and refuse to communicate.

\section{Design Strategy of Cognitive Training System Based on SCD Elderly's Behavioral and Psychological Characteristics}

The existing cognitive training systems are all PC-based platforms. The keyboard and mouse are used for cognitive training, but due to the fact that most elderly people do not have the habit of using computers frequently or cannot use computers and the complexity of computer operation, keyboard and mouse operation requires a long period of adaptation.

The cognitive training system conceived and designed in this paper is based on Android tablet. At present, most elderly people use smart phones. Tablet operation is closer to mobile phone operation, which will reduce the adaptation time of users. The screen size of the mobile phone is too small for the aged with poor eyesight to see clearly.

The functional design of the cognitive training system should include the popularization of AD-related knowledge, the cognitive training planned by the system, the generation of reports after training and the close contact with doctors.

\subsection{Content Design Strategy Based on SCD Elderly's Behavioral and Psychological Characteristics}

(1) Popularize AD-related information and rehabilitation knowledge

Due to the lack of knowledge about AD among the elderly in our country, most patients regard the early symptoms of $\mathrm{AD}$ as normal functional degeneration of the elderly and show indifference, which may delay the golden stage of effective intervention and prevention of 
AD. However, some patients know that they are in the early stage of $\mathrm{AD}$, but do not know the specific methods to intervene and prevent AD. It is necessary to let the elderly know the pathological knowledge and rehabilitation knowledge of AD. But the most important thing is for caregivers who care for patients, so that caregivers can also understand the pathology of AD, how to prevent and treat $\mathrm{AD}$, specific intervention methods and how to regulate the care of patients.

(2) Combining cognition with outdoor training

1) Intensive training; After the first cognitive training, a training report will be generated in the report column. The results of each training are bar charts, which clearly and intuitively show the weak items and strong points of the user's training. Intensive training will be conducted for the cognitive domain corresponding to the weak items, and periodic training will be set up. One week will be taken as the training cycle, and the training of the weak items will be strengthened in combination with the generated report forms.

2) The combination of cognitive training and outdoor exercise; At the same time of cognitive training, the patient's physical health is also a top priority. The combination of exercise training and cognitive training can ensure the patient's internal and external health. Organize patients to do outdoor exercises as much as possible. For example: Tai Chi, aerobic exercise, cycling, walking on treadmill, elliptical motion; outdoor walking, etc. To choose outdoor activities with different degrees according to the patient's physical condition.

3) 'One Person, One Policy'Training Plan

Due to the individual differences of patients' cognitive impairment types, the cognitive training combination of each patient is different due to the different cognitive impairment types and the different degree of decline of each patient and varies from person to person. If only memory declines, emphasis should be placed on strengthening memory training. If there is a deterioration in memory and calculation ability, a combination of memory and calculation ability will be trained. According to the difference of disease types, the system can suit the remedy to the case and strike steadily.

4) Strengthen the relationship among doctors, patients and caregivers

The treatment mode of doctor-patient combination can effectively strengthen the connection between patients and doctors. Doctors can understand the patient's condition and recovery progress in real time. However, strengthening the combination of medical care can enable caregivers to carry out more standardized care for patients. Caregivers report the current situation of patients to doctors in real time to help doctors implement treatment plans. Patients are closely related to caregivers. caregivers should not only take good care of the patients' diet and daily life and implement the training plan, but the most important thing is to understand the patients' psychological changes, eliminate the patients' negative mentality, encourage the patients, do not abandon and do not give up.

The system establishes patient end and doctor end respectively. Patient end trains patients and records training details. The doctor end facilitates the doctor to supervise the illness in real time in case of delay. According to the reports received, doctors know the current situation of patients, describe the patient's condition to caregivers, and change the training program at any time. The caregiver implements the patient's training tasks followed by the doctor's advice.

\subsection{Interactive Design Strategy Based on SCD Elderly's Behavioral and Psychological Characteristics}

(1) Based on the principle of interaction design, an interaction design strategy that conforms to the behavioral and psychological characteristics of SCD elderly is designed:

1) System Visibility; Elderly people should know their current position and state of the system and give the elderly appropriate feedback when operating the cognitive training system. If the operation fails, timely feedback should be given to the old man to indicate the operation failure; When there is a bug, you should also give a bug prompt in time; When the reaction time is too long, use the form of a progress bar to inform the elderly of the current situation.

2) Matching System and Real World; Close to the cognition of the elderly, the icons and terms used should be within the understanding range of the elderly, and the situation should be described in the simplest and straightforward language.

3) The Controllability and Freedom of Users; For the elderly's misoperation, secondary confirmation or cancellation orders should be provided to improve the controllability of the elderly's operation.

4) Consistency; Unified system of copywriting, visual style, component style, etc.; Keep the system language, function and operation consistent, so that the old people are familiar with the system.

5) Error Proofing; Gray or hide operations that the elderly cannot perform to reduce the elderly's wrong operations.

6) Recognition is Better than Memory; To reduce the memory load of the elderly, actions and options should be visible. The elderly people don't have to remember all the information, the instructions of the system should be visible or easily accessible.

7) Flexible and Efficient; Ensure that the cognitive training system can enable the elderly to get started quickly and without complexity.

8) Aesthetic and Simple Design; Clear distinction between light and heavy, highlighting important functions, content and clear layout content, so that the elderly at a glance, convenient memory.

9) Fault Tolerance; Error information should not be displayed by code but expressed by language. The problem should be accurately fed back to the elderly and solutions should be put forward to the elderly.

10) Assistance Document; Set up assistance document to help the elderly clearly understand a concept in the system and help the elderly to better solve problems.

(2) Using Graphic Elements to Set up Guidance Functions 
Considering that patient side is shared by patients and caregivers, default options are set according to user roles. When entering the system, graphic elements and two personal graphic options are used to visually distinguish the caregiver mode from the elderly mode. The novice guidance setting is an important link to guide users to understand and identify the system functions. The caregiver mode only needs normal first guidance. The guidance function is set as the default in the elderly mode. The guidance function is accepted every time they enter the system, so that the elderly will not bother to remember each function.

(3) Easily Recognized Interface Design

As the elderly grow older, it is difficult to recognize various colors visually. However, the SCD elderly recognition ability for cold colors such as purple, blue and green decreases. White is easily mistaken for yellow. In the visual interface design of the elderly, bright colors and long-wave contrasting colors should be selected, with warm colors as the main color system, such as yellow, orange, red, etc. to divide the functional areas, so as to facilitate the elderly to quickly recognize the interface function division. In the visual mode, the elderly should be guided by the combination of easily recognizable graphics or real pictures and characters familiar to the elderly, so that the elderly can acquire information faster. The training environment should take the real life scenes related to the elderly or scenes designed with real scenes as elements as the main scenes, so that the elderly can respond to the situations in the scenes more quickly and effectively. Based on this, several key points of the cognitive training system interface can be summarized:

1) The color of the system interface tends to be bright color and long-wave contrast color.

2) System layering should be simple, concise and clear.

3) The shapes of the icons are mostly soft lines and obtuse angles without pressure.

4) Due to the visual characteristics of the elderly, the layout of the interface picture and text should conform to the visual characteristics of the elderly so that the elderly can see clearly.

\section{Conclusion}

Based on the behavioral and psychological characteristics of SCD elderly, the content strategy of cognitive training system is obtained. Combined with interaction design principles, the interaction strategy of cognitive training system is presented. The system should be close to the characteristics of the SCD elderly, solve problems for the SCD elderly, and improve the goodwill of the SCD elderly towards the system. Due to the cognitive training system is relatively new in China, and the continuous development of SCD research and the limitations of literature research, this article may not be very comprehensive and perfect. These design strategies may vary with the actual design. In addition, as far as our national conditions are concerned, the living habits and educational level of each elderly person are different. Therefore, more research is needed to adjust and perfect the design scheme.

\section{Acknowledgments}

This paper was supported by Chinese Ministry of education of Humanities and Social Science (project No. 17YJAZH100).

\section{Author's brief introduction}

Xue Yanmin, female, Han nationality, native of Henan, professor and doctor of industrial design department of $\mathrm{Xi}^{\prime}$ an university of technology, whose main research direction is ergonomics design. Tel: $13571939092 \mathrm{E}$-mail: 915728096@qq. Com

Lin Rui, male, Han nationality, native of Fujian, is a master's student in the Department of Industrial Design of Xi 'an University of Technology. Tel: 13193374767 Email: 736996903@qq.Com

Ge Chang, female, Han nationality, native of Henan, lecturer and doctor in the department of industrial design of Xi 'an university of technology, whose main research direction is product innovation design. Tel: 13572882660 E-mail: 81762598@qq.com

\section{References}

1. Hou Yiyi. Design of Rehabilitation Toys for Senile Patients with Alzheimer's Disease [J] . Journal of Shandong Institute of Arts and Crafts.2014.(02) :5456.

2. Sperling R A,Al E.Toward Defining the Preclinical Stages of Alzheimer's Disease: Recommendations from the National Institute on Aging-Alzheimer's Association Workgroups on Diagnostic Guidelines for Alzheimer's Disease.[J]. Alzheimer's \& Dementia : The Journal of the Alzheimer's Association. 7. 2011 May.7(3) :280-92.

3. Reisberg B, Gauthier S. Current Evidence for Subjective Cognitive Impairment (SCI) as the Premild Cognitive Impairment (MCI) Stage of Subsequent Manifest Alzheimer's Disease[J]. International Psychogeriatrics.2008. 20(1) :1-16.

4. Gauthier S, Reisberg B, Zaudig M, etc. (2006) Mild Cognitive Impairment. Lancet 367: 1262-1270.

5. Jessen F,etc.A conceptual framework for research on subjective cognitive decline in preclinical Alzheimer|"s disease[J] . Alzheimer|"s \& Dementia, 2014. 10(6):844-852.

6. Clare L. Cognitive training and cognitive rehabilitation for people with early-stage dementia[J]. Reviews in Clinical Gerontology.2003.13(01) :75-83.

7. Clare L, Woods R T . Cognitive training and cognitive rehabilitation for people with early-stage Alzheimer's disease: A review[J]. Neuropsychological Rehabilitation. 2004.14(4) :385-401.

8. Han Ying. Diagnosis and Treatment Strategies for Subjective Cognitive Decline in Preclinical Alzheimer's Disease in China $[\mathrm{J}]$. Chinese Journal of Clinical Medical Imaging.2018.v.29(08) :8-12.

9. Fernández-Blázquez, Miguel A, ávila-Villanueva, 
Marina, Maestú, Fernando, et al. Specific Features of Subjective Cognitive Decline Predict Faster Conversion to Mild Cognitive Impairment [J] . Journal of Alzheimerl"s Disease.2016.52(1):271-281.

10. Wei Fangzhou. Interactive Design of Cognitive Training System for Elderly People with Mild Cognitive Impairment [D] . 2017.

11. Zhu Lin, Song Weiqun, Yue Yuehong, et al. Effects of Computer-assisted Cognitive Training on Cognitive Function and Depression in Patients with Brain Injury [J] . Chinese Journal of Cerebrovascular Diseases. 2011. 08(10):508-512.

12. Wang Xiaona,etc. Observation on the Effect of Computer Assisted Cognitive Rehabilitation System on Cognitive Impairment after Stroke [J] . China Rehabilitation. 2013(5) :330-332.

13. "Basic Cognitive Ability Test" (Version 2.0) and Brief Introduction of Its Software [J]. Advances in Psychological Science (6) :874.

14. Reisberg B, etc.The pre-mild cognitive impairment, subjective cognitive impairment stage of Alzheimer's disease.[J].Alzheimer's \& dementia : the journal of the Alzheimer's Association,2008,4(1 Suppl 1) :S98S108. 